\section{Hipercalcemia severa en el curso de rabdomiolisis: caso clínico}

\author{
JUAN PABLO HUIDOBRO E. ${ }^{1}$, JAIME SANTANDER ${ }^{2}$, \\ DANIEL VICENTINI $^{3}$, AQUILES JARA ${ }^{1}$
}

\section{Rhabdomyolysis and severe hypercalcemia. Report of one case}

Rhabdomyolysis (RD) is the process that leads to cell destruction of striated muscle. Causes include inherited metabolic defects or acquired disorders. RD is frequently associated with acute kidney injury (AKI) and disorders of calcium metabolism. We report a 33 year old man that after amphetamine consumption and an uninterrupted 3,000 km driving presented vomiting, muscle pain and dark urine. He had elevated creatinkinase levels, severe hypocalcemia and an acute renal failure. He was treated with hemodialysis and calcitriol. He was transferred to our hospital and on admission a serum calcium of $18 \mathrm{mg} / \mathrm{dl}$ was detected. He continued on hemodialysis, recovering renal function and with normalization of creatinkinase levels and serum calcium level.

(Rev Med Chile 2019; 147: 125-129)

Key words: Acute Kidney Injury; Hypercalcemia; Rhabdomyolysis.
'Departamento de Nefrología. Escuela de Medicina, Pontificia Universidad Católica de Chile. Santiago, Chile.

${ }^{2}$ Departamento de Psiquiatría. Escuela de Medicina, Pontificia Universidad Católica de Chile. Santiago, Chile.

${ }^{3}$ Servicio de Medicina Nuclear. Escuela de Medicina, Pontificia Universidad Católica de Chile. Santiago, Chile.

Trabajo no recibió financiamiento. Los autores declaran no tener conflictos de interés.

Recibido el 10 de octubre de 2018, aceptado el 29 de noviembre de 2018.

Correspondencia a: Dr. Aquiles Jara Departamento de Nefrología Diagonal Paraguay 362, piso 4 Santiago, Chile.

ajara@med.puc.cl
L a rabdomiolisis $(\mathrm{RD})$ es el proceso que lleva a la necrosis celular del músculo estriado, con daño de la integridad de la membrana plasmática y la liberación de componentes intracelulares al torrente sanguíneo. Las causas más frecuentes son trauma, ejercicio extremo, isquemia y uso de drogas. Otras etiologías menos frecuentes son convulsiones, infecciones y miotoxinas ${ }^{1}$. El desarrollo de injuria renal aguda (IRA) en la RD aparece en 15-50\% de los casos, generalmente asociado a depleción de volumen. En ocasiones puede acompañarse de alteraciones del metabolismo del calcio, los que habitualmente ocurren con un patrón bifásico. En la fase inicial oligúrica, $60 \%$ de los pacientes con RD puede presentar hipocalcemia ${ }^{2}$. Esta ocurre como consecuencia de la precipitación de calcio con el fósforo liberado de la célula muscular dañada. En la fase de recuperación del daño renal, $20-30 \%$ pueden desarrollar hipercalcemia ${ }^{3,4}$, principalmente como resultado de la movilización de los depósitos de calcio-fósforo, más que por exceso de PTH o vitamina $\mathrm{D}^{5}$. La hipercalcemia en la fase de recuperación de la IRA en RD no es un fenómeno infrecuente, aunque por lo general es de leve cuantía y dura pocos días. La hipercalcemia severa es infrecuente en el curso de una RD, existiendo solo 5 casos publicados con calcemia mayor a $15 \mathrm{mg} / \mathrm{dL}$.

Comunicamos un caso de rabdomiolisis que desarrolló IRA con requerimientos de hemodiálisis y trastorno de la calcemia severo, con fase inicial hipocalcémica y fase posterior hipercalcémica con valores de calcio plasmático hasta $18,2 \mathrm{mg} / \mathrm{dL}$.

\section{Caso clínico}

Hombre de 33 años, con antecedentes de atrofia marcada de riñón izquierdo, función renal normal y enfermedad bipolar en tratamiento psiquiátrico, que semanas antes de su historia actual había suspendido toda medicación. Tres semanas antes de su ingreso, estando en el extranjero, usó drogas estimulantes del tipo anfetaminas, posterior a lo cual condujo en automóvil, sin pausas ni ingesta apropiada de líquidos, por más de 3.000 ki- 
lómetros. Posterior a este esfuerzo físico comenzó con compromiso del estado general, dolores musculares principalmente en muslo derecho, vómitos y orinas oscuras, seguidas de oligoanuria. Fue visto inicialmente en un servicio de urgencia ambulatorio, donde se le indicó analgesia y antieméticos. Por empeoramiento de su sintomatología consultó en un hospital, donde se realizaron exámenes de laboratorio en los que destacó creatinfosfoquinasa (CPK) elevada e insuficiencia renal aguda acompañada de hipocalcemia severa (Tabla 1). Se inició hemodiálisis y se indicó calcitriol 0,25 ug/día. Dos semanas después, el paciente fue trasladado en avión-ambulancia a nuestro centro, donde ingresó vigil, inquieto, con dolor en extremidades inferiores, nauseoso e hipertenso moderado. Había recuperado diuresis (alrededor de 1 litro al día) y al examen físico destacaba ausencia de congestión pulmonar y empastamiento muscular al examen de extremidades inferiores. Resto de la exploración fue normal. En exámenes de ingreso se constató hipercalcemia severa con calcio total $18,2 \mathrm{mg} / \mathrm{dl}$ y calcio iónico de 7,1 mg/dl. Los niveles séricos de hormona paratiroidea $(\mathrm{PTH})$ fueron de
$9 \mathrm{pg} / \mathrm{ml}$, y los de 25-hidroxivitamina D de $10 \mathrm{ng} /$ $\mathrm{ml}$. Una ecografía doppler renal mostró riñón derecho de $17,8 \mathrm{~cm}$ en su eje longitudinal mayor, con leve hiperecogenicidad, índice de resistencia intrarrenal de 7,2, sin dilatación pielocaliciaria ni evidencias de nefrocalcinosis, y riñón izquierdo atrófico, de 4,6 cm, con marcada disminución del espesor cortical y dilatación pielocaliciaria. Un cintigrama óseo con $\mathrm{Tc}^{99 \mathrm{~m}}$-MDP reveló extensa captación muscular difusa de radiofármaco en el cuello, pared abdominal y grupos musculares proximales de extremidades superiores e inferiores. Había además intensa captación pulmonar y gástrica difusa (Figura 1). Continuó con sesiones de hemodiálisis según requerimientos con descenso progresivo de calcio plasmático y retención nitrogenada (Tabla 2). Posteriormente cursó con fase poliúrica de recuperación IRA con diuresis alrededor de 7 litros al día. Presentó además mejoría clínica y analítica con descenso de CPK a valores normales. Al alta, la creatinina fue de $1,5 \mathrm{mg} / \mathrm{dl}$, CPK normal y Ca sérico: 9,6 mg/dl. Diez meses después del inicio de sus síntomas la creatininemia fue de $1,16 \mathrm{mg} / \mathrm{dl}$ y calcemia de $9,8 \mathrm{mg} / \mathrm{dl}$.

Tabla 1. Niveles séricos al ingreso a hospital en Estados Unidos y a nuestro hospital (2 semanas después)

\begin{tabular}{|c|c|c|}
\hline Niveles séricos & Ingreso hospital EE.UU. & Ingreso a nuestro hospital \\
\hline Sodio (mmol/L) & 125 & 136 \\
\hline Potasio (mmol/L) & 6,0 & 5,8 \\
\hline Cloro (mmol/L) & 88 & 93 \\
\hline Bicarbonato (mmol/L) & 16 & 27 \\
\hline Calcio total (mg/dL) & 5,8 & 15,2 \\
\hline Calcio iónico (mg/dL) & No medido & 7,1 \\
\hline Fósforo (mg/dL) & 8,4 & 8,5 \\
\hline Albúmina (g/dL) & 2,5 & 3,3 \\
\hline Creatinina (mg/dL) & 21,3 & 9,7 \\
\hline Nitrógeno ureico (mg/dL) & 162 & 55 \\
\hline Lactato deshidrogenasa (U/L) & No medida & 351 \\
\hline Ácido úrico (mg/dL) & No medida & 7,0 \\
\hline Magnesio (mg/dL) & 2,8 & 2,2 \\
\hline CPK (U/L) & 35.966 & 225 \\
\hline
\end{tabular}




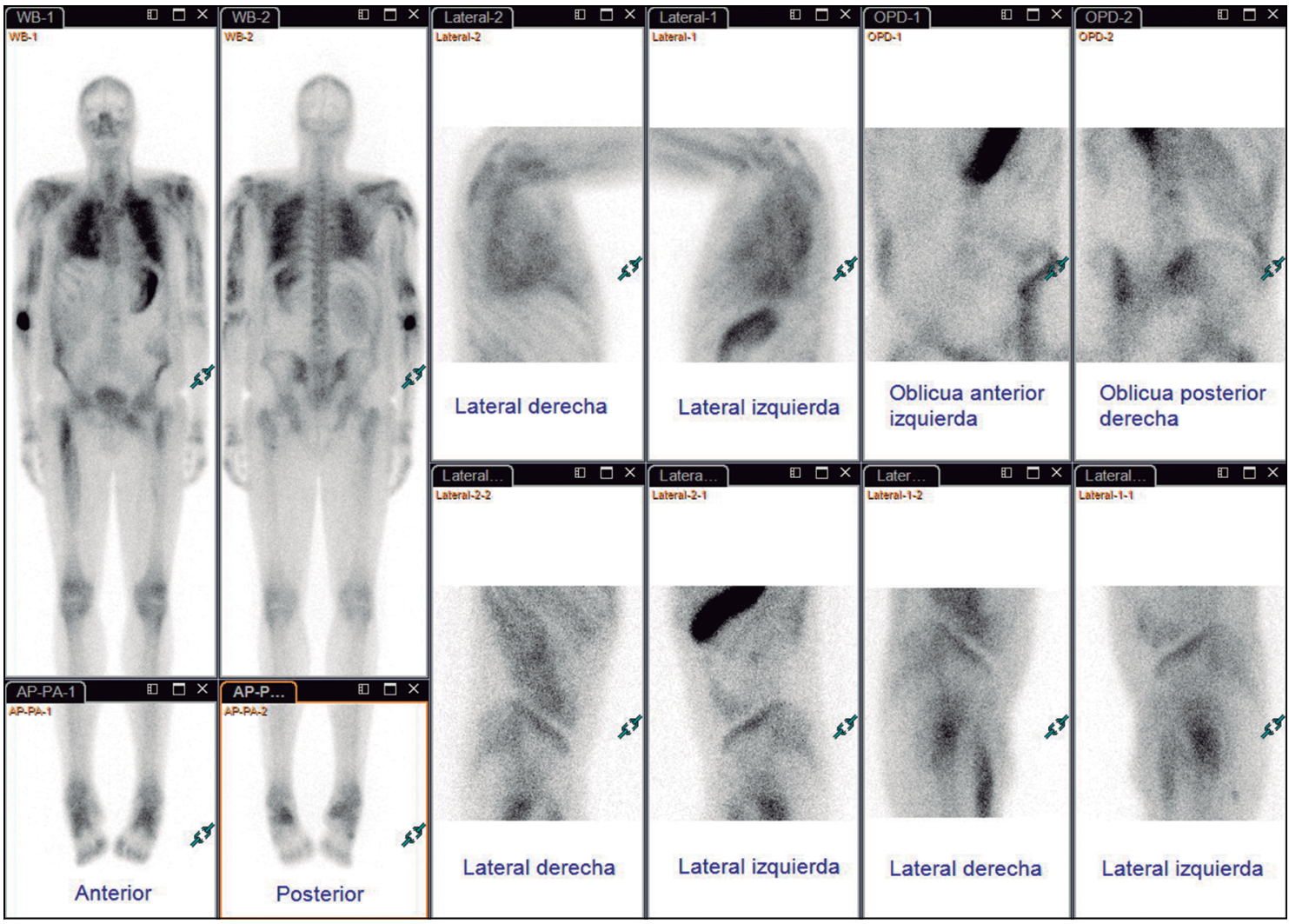

Figura 1. Cintigrama óseo con Tc99m-MDP.

Tabla 2. Evolución de exámenes durante la estadía en nuestro hospital

\begin{tabular}{|c|c|c|c|c|c|c|c|c|c|c|c|c|c|}
\hline $\begin{array}{l}\text { Parámetro/día de } \\
\text { hospitalización }\end{array}$ & $1 *$ & 2 & $3 *$ & $4^{*}$ & $5^{*}$ & 6 & 7 & 8 & 10 & 11 & 12 & 13 & 16 \\
\hline Calcemia (mg/dL) & 15,2 & 14,7 & 18,2 & 13,6 & 13,6 & 12 & 11,8 & 11,2 & 10,7 & & 8,5 & & 9,6 \\
\hline Calcio iónico (mg/dL) & 7,1 & & 6,9 & & & & & & & & & & \\
\hline CPK (U/L) & 225 & 124 & 119 & 142 & & & & & 205 & 188 & 183 & & \\
\hline $\mathrm{LDH}(\mathrm{U} / \mathrm{L})$ & 304 & & 351 & & & & 230 & & & & & & \\
\hline Creatinina (mg/dL) & 9,7 & 8,3 & 9,9 & 7,5 & 6,2 & 5,27 & 4,21 & 3,72 & 3,65 & 2,98 & 2,2 & 1,8 & 1,5 \\
\hline Nitrógeno ureico (mg/dL) & 55 & 49 & 65 & & & 33 & 36 & 30 & 53 & 54 & 44 & & 30 \\
\hline Sodio (mmol/L) & 136 & 131 & 129 & 128 & 138 & 138 & 138 & 139 & 142 & 142 & 141 & 150 & 138 \\
\hline Potasio (mmol/L) & 5,8 & 6,2 & 6,1 & 5,9 & 4,7 & 4,3 & 4,0 & 4,0 & 4,9 & 5,1 & 4,1 & 4,9 & 5,2 \\
\hline Fósforo (mg/dL) & 8,4 & & 8,4 & 7,5 & 7,7 & 6,7 & 4,73 & 3,83 & 4,68 & & 3,7 & & 4 \\
\hline Albúmina (g/dL) & 3,3 & & 3,2 & & & & 3,4 & 3,6 & 4,4 & & & & \\
\hline Ácido úrico (mg/dL) & 5,5 & & 7,0 & & & & 4,0 & & 6,4 & & & & \\
\hline Hematocrito (\%) & 30,9 & 31,7 & & & & & & & & & & 31,4 & \\
\hline $25-\mathrm{OH}$ vitamina $\mathrm{D}(\mathrm{ng} / \mathrm{mL})$ & & & 10,4 & & & & & & & & & & \\
\hline PTH (pg/mL) & & & 9 & & & & & & & & & & \\
\hline Diuresis $(\mathrm{mL})$ & 1.000 & & & & & & & & & 10.500 & & & \\
\hline
\end{tabular}

*En asterisco se indican los días en que se realizó hemodiálisis. 


\section{Discusión}

La rabdomiolisis ocurre cuando una injuria a la célula muscular gatilla una serie de eventos que determinan una alteración progresiva en la homeostasis intracelular de calcio y, finalmente, la muerte celular. Normalmente, la concentración de calcio citoplasmática es estrictamente regulada por varios transportadores y bombas de calcio (canales de calcio, transportador $2 \mathrm{Na}^{+}$/ $\mathrm{Ca}^{++}$y la bomba Ca ${ }^{2+}$ ATPasa). El calcio es transportado desde el sistema reticulosarcoplásmico vía la bomba $\mathrm{Ca}^{2+} \mathrm{ATPasa}$ donde es liberado por estimulación neural para causar contracción muscular. El calcio es bombeado desde el citoplasma al espacio extracelular a través del transportador $2 \mathrm{Na}^{+} / \mathrm{Ca}^{++}$moviendo $\mathrm{Na}^{+}$a favor de su gradiente de concentración, mientras mueve $\mathrm{Ca}^{++}$en contra de gradiente. El resultado neto es que la concentración de calcio ionizado intracelular es 10.000 veces más baja que la concentración de calcio ionizado extracelular ${ }^{1,2}$.

En la rabdomiolisis los dos mecanismos que gatillan la cascada de eventos que resultan en muerte celular son: 1) pérdida de ATP, lo que causa disfunción de la $\mathrm{Na}^{+} / \mathrm{K}^{+}$ATPasa y la $\mathrm{Ca}^{++} \mathrm{ATPa}-$ sa, determinando acumulación de calcio citosólico, y 2) la pérdida de integridad de la membrana del sistema reticulosarcoplásmico, lo que también induce aumento de la concentración de calcio citosólico. El aumento del calcio citosólico activa proteasas neutrales de fosfolipasas lo que daña las membranas celulares e induce la liberación de contenido intracelular al extracelular. La liberación de este contenido intracelular, que incluye potasio, fósforo, ácido úrico y creatininakinasas, puede llevar a hiperkalemia significativa con riesgo de arritmias y a hiperfosfemia, que causará hipocalcemia debido a la formación de depósitos de calcio-fosfato en los músculos dañados, tal como vimos en nuestro paciente. Durante la fase de recuperación de la $\mathrm{RD}$ los depósitos de calcio-fosfato se movilizan causando hipercalcemia que puede ser severa como en este caso ${ }^{1}$.

En la literatura, desde 1970, se encuentran publicados más de 60 casos de hipercalcemia asociada a $\mathrm{RD}^{5-11}$. Es destacable que la mayoría de los casos descritos de hipercalcemia mayor de 15 $\mathrm{mg} / \mathrm{dl}$, como el del paciente que exponemos, presentan inicialmente una hipocalcemia marcada ${ }^{5-9}$. Nuestro paciente presentó hipocalcemia de 5,1 $\mathrm{mg} / \mathrm{dl}$ en la fase oligoanúrica y posteriormente una hipercalcemia de 18,2 mg/dl. Grobety y cols., describieron dos casos de pacientes con hipocalcemia leve en la fase inicial, de 7,4 mg/dl y 7,6 mg/dl, que posteriormente desarrollaron una hipercalcemia grave de $15,6 \mathrm{mg} / \mathrm{dl}$ y $15 \mathrm{mg} / \mathrm{dl}$, respectivamente ${ }^{11}$. El nivel de calcio plasmático más elevado en $\mathrm{RD}$ citado en la literatura es de $20 \mathrm{mg} / \mathrm{dl}$. Hay otros 5 casos de hipercalcemia con niveles $>15 \mathrm{mg} / \mathrm{dl}$. En los últimos 20 años la duración de hipercalcemia comunicada ha sido en promedio 8,6 días con una mediana de 10 días.

La hipocalcemia en la RD suele aparecer en la fase oligúrica de la IRA, normalmente es transitoria y excepcionalmente es severa y sintomática. El tratamiento con sales de calcio y/o derivados de la vitamina $\mathrm{D}$ se debe evitar, dada la potencialidad de agravar la hipercalcemia propia de la fase de recuperación de la $\mathrm{RD}^{5}$. Nuestro paciente recibió calcitriol en la fase inicial de su trastorno.

La hipercalcemia en la RD suele ocurrir en la fase de recuperación de la IRA y se debe principalmente a la liberación del calcio depositado en los músculos a la circulación sanguínea, además de un hiperparatiroidismo leve en el marco de la insuficiencia renal ${ }^{4,5}$. La mayoría de los casos de hipercalcemia son autolimitados y solo requieren observación e hidratación adecuada. Sin embargo, ciertos casos pueden requerir tratamiento con bifosfonatos, calcitonina y diuréticos de asa ${ }^{4}$.

Nuestro paciente tuvo niveles de PTH elevados al ingreso, asociados a una hipocalcemia marcada, mientras que en la fase de recuperación de la diuresis presentó hipercalcemia severa con PTH suprimida y niveles de calcidiol bajos, como describen otros autores ${ }^{5}$. En la fase de recuperación de su IRA presentó poliuria marcada y normalización progresiva de parámetros nitrogenados y trastornos metabólicos, lo que permitió la discontinuación de hemodiálisis.

\section{Conclusiones}

La ocurrencia de injuria renal aguda es frecuente en el contexto de rabdomiolisis y se acompaña frecuentemente de alteraciones en la cinética del calcio.

Ante la presencia de IRA secundaria a RD, los niveles plasmáticos de calcio deben ser estrictamente monitorizados, dado que una hipocalcemia 
inicial puede preceder la ocurrencia de hipercalcemia severa durante la fase de recuperación de la IRA. Por lo mismo, la suplementación de calcio $\mathrm{y}$ el aporte de derivados de vitamina $\mathrm{D}$ deben restringirse a situaciones de hipocalcemia severa, a fin de evitar una hipercalcemia severa de rebote.

\section{Referencias}

1. Giannoglou GD, Chatzizisis YS, Misirli G: The syndrome of rhabdomyolysis: Pathophysiology and diagnosis. Eur J Intern Med 2007; 18 (2): 90-100.

2. Bosch X, Poch E, Grau JM. Rhabdomyolysis and acute kidney injury. N Engl J Med 2009; 361: 62-72.

3. Cetinkaya R, Uyanik A, Keles M, Bilen Y. The rhabdomyolysis related acute renal failure and biphasic calcium metabolism. Pak J Med Sci 2009; 25 (1): 152-4.

4. Akmal M, Bishop JE, Telfer N, Norman AW, Massry SG. Hypocalcemia and hypercalcemia in patients with rhabdomyolysis with and without acute renal failure. J Clin Endocrinol Metab 1986; 63 (1): 137-42.

5. Shrestha SM, Berry JL, Davies M, Ballardie FW. Biphasic hypercalcemia in severe rhabdomyolysis: Serial analysis PTH and vitamin D metabolites. A case report and literature review. Am J Kidney Dis 2004; 43: e315.

6. Kumar R, West DM, Jingree M, Laurence AS. Unusual consequences of heroin overdose: rhabdomyolysis, acute renal failure, paraplegia and hypercalcaemia. $\mathrm{Br} \mathrm{J}$ Anaesth 1999; 83 (3): 496-8.

7. Sperling LS, Tumlin JA. Delayed hypercalcemia after rhabdomyolysis- induced acute renal failure. Am J Med Sci 1996; 311 (4): 186-8.

8. Feinstein EI, Akmal M, Telfer N, Massary SG. Delayed hypercalcemia with acute renal failure associated with nontraumatic rhabdomyolysis. Arch Intern Med 1981; 141 (6): 753-5.

9. Graziani G, Calvetta A, Cucchiari D, Valaperta S, Montanelli A. Life-threatening hypercalcemia in patients with rhabdomyolysis-induced oliguric acute renal failure. J Nephrol 2011; 24 (1): 128-31.

10. Zerr B, Durand C, Ceccoli A, Fogliani J. Severe hypercalcemia after rhabdomyolysis and acute renal failure. Ann Fr Anesth Reanim 1991; 10: 482-5.

11. Grobéty M, Rosman J, Schaller MD, Wauters JP, Perret C. Rhabdomyolysis, acute renal insufficiency and hypercalcemia. Schweiz Med Wochenschr 1992; 122 (29): 1087-90. 